\title{
ATPasa de calcio en el sistema nervioso
}

\author{
Myriam L. Velandia ${ }^{1}$, Zayra V. Garavito ${ }^{1}$, María L. Caldas ${ }^{2}$, Hernán Hurtado ${ }^{1}$ \\ ${ }^{1}$ Laboratorio de Neurociencias, Instituto Nacional de Salud, Bogotá, D.C., Colombia. \\ ${ }^{2}$ Unidad de Microscopía y Análisis de Imagen. Instituto Nacional de Salud, Bogotá, D.C., Colombia. \\ La bomba de calcio es una proteína integral de la membrana que regula el calcio libre \\ citoplasmático en concentraciones menores de $0,1 \mathrm{mM}$. En la mayoría de las células eucarióticas \\ está ubicada en la membrana, en el plasmalema o en organelos como el retículo \\ sarcoendoplásmico y los calciosomas. Su actividad está dada por la hidrólisis del ATP, la \\ concentración del ion en el citoplasma y por otros factores que la regulan como la calmodulina, \\ los fosfolípidos y las proteínas-cinasas. Por diferentes métodos, se ha detectado la ATPasa de \\ calcio y su actividad tanto en tejido nervioso central y periférico, como en otros tejidos.
}

Palabras clave: calcio, ATPasa de calcio, citoquímica enzimática, sistema nervioso.

\section{Calcium ATPase in the nervous system}

The calcium pump is an integral membrane protein, which regulates free citoplasmatic calcium using ATP energy. It controls citoplasmatic calcium concentrations below $0.1 \mathrm{mM}$. It is located in all eucariotic cells in plasmalemma, sarcoendoplasmic reticulum and calciosomes. Its activity depends on ionic concentration, ATP hydrolysis and other regulators such as calmodulin, phospholipid and protein-kinases. This enzyme and its activity has been detected through several methods in the central nervous system as well as in other tissues.

Key words: calcium, calcium ATPase, enzymatic cytochemistry, nervous system.

En la mayoría de las células, la concentración interna de calcio es de $0,1 \mathrm{mM}$ y la externa de 1,0 $\mathrm{mM}$ (1). Cuando existe un estímulo químico o eléctrico en las células, el calcio ingresa al citoplasma a través de canales, algunos voltajedependientes o activados por ligandos, ubicados sobre la membrana plasmática (2). Por propiedades físicas del ion, como su radio atómico y sus cargas positivas, éste se une específicamente a proteínas dependientes de él y desencadena respuestas dentro de la célula, tales como la activación de rutas metabólicas, la liberación de neurotransmisores y la síntesis y activación de proteínas, entre otras. Por estas razones, se considera como un ion mensajero (1-3).

Después de su ingreso, la célula regula la concentración del ion, captándolo por medio de

Correspondencia:

mcaldas@hemagogus.ins.gov.co

Recibido: 07/03/00; aceptado: 17/11/00 proteínas como la calmodulina, la parvoalbúmina y la troponina $\mathrm{C}$, entre otras $(4,5)$; también puede ser almacenado en organelos especializados o expulsado de la célula. Se conocen dos sistemas a través de los cuales se realiza este último proceso en las células: uno, de intercambio, en el cual se expulsa calcio y entra sodio impulsado por la energía electroquímica generada por el flujo a favor del gradiente de concentración del sodio $(2,6)$; el segundo es un sistema de transporte activo en el que se utiliza la energía liberada por la hidrólisis del ATP para expulsar el ion al espacio extracelular. La proteína que se encarga de esta función se denomina ATPasa de calcio $(7,8)$, la cual actúa con mayor velocidad y afinidad al ion que el sistema de intercambio y que fue descubierta inicialmente en eritrocitos $(9,10)$.

Esta ATPasa se clasifica como una enzima hidrolítica de tipo $\mathrm{P}$ por la presencia de un residuo aspartil al cual se une el Pi proveniente de la hidrólisis del ATP, y forma un intermediario 
fosforilado que le suministra la energía para sus cambios de conformación.

Por diferentes metodologías se ha podido detectar la presencia y la actividad de la ATPasa en los diferentes tejidos. En el sistema nervioso periférico, por ejemplo, se han utilizado técnicas inmunocitoquímicas para comprobar la presencia de algunas isoformas de la enzima. Por otro lado, mediante citoquímica enzimática, se ha detectado su acción catalítica y se ha encontrado actividad sobre la membrana plasmática de células gliales y axones, incluidos algunos organelos como el retículo endoplásmico (11).

Esta revisión tiene por objeto divulgar aspectos generales y resumir lo encontrado en la literatura acerca de la estructura y función de la ATPasa de calcio, con el interés especial de concentrar la escasa información relacionada con el sistema nervioso periférico y el papel que desempeña esta enzima en los diferentes procesos que involucra un nervio lesionado.

\section{Generalidades del calcio en las células}

Todas las células necesitan de un ambiente iónico que les permita mantener un equilibrio entre el medio externo e interno (12). Uno de los iones importantes en este ambiente iónico es el calcio (13), el cual se encuentra normalmente en mayor concentración en el medio extracelular. Cuando la célula es estimulada eléctricamente (impulso nervioso) o químicamente (por medio de hormonas o neurotransmisores), el calcio ingresa a la célula, a favor de su gradiente de concentración, generando fluctuaciones en la concentración interna del calcio que oscilan entre 0,1 y $0,2 \mathrm{mM}$. Estas fluctuaciones en la concentración del ion en las células son 'mensajes' que deben ser traducidos y sobre los cuales la célula genera alguna respuesta (14).

Esta efectividad del calcio como ion mensajero se debe a la presencia en el citoplasma de proteínas que captan las variaciones en su concentración intracelular y lo capturan. Estas proteínas especializadas pertenecen en su mayoría a la superfamilia EF-hands, debido a que poseen una o más zonas denominadas bucles o asas. El calcio se une en estas zonas específicamente, generando cambios en la conformación de la proteína receptora, traduciendo el mensaje y generando respuesta, que puede ser, como en el caso de la calmodulina, el ensamblaje y desensamblaje de tubulina o la síntesis de proteínas $(5,14)$. Se cree que estos fenómenos mediados por el calcio son semejantes en todas las células; sin embargo, el calcio tiene gran importancia en las células excitables como son las musculares y nerviosas.

En el tejido nervioso, por ejemplo, el calcio participa normalmente en funciones como el transporte axonal anterógrado, en el reciclaje de substancias (15-17) y en la liberación de neurotransmisores de las vesículas sinápticas. Esto último lo realiza despolarizando la membrana presináptica, al desencadenar la liberación exocítica de los neurotransmisores (18).

En el sistema nervioso central, algunos estados patológicos como la anoxia y la isquemia son mediados, principalmente, por un aumento en la concentración de calcio libre citoplasmático, lo cual induce muerte celular (19).

Se sabe que en el sistema nervioso periférico los cambios morfológicos debidos a la transección del nervio y posterior degeneración del segmento distal, proceso conocido como degeneración walleriana, están dados, entre otros factores, por un aumento en la concentración de calcio libre, activando proteasas que conducen a la desintegración granular del citoplasma y al desensamblaje de neurofilamentos $(15,20,21)$.

Mediante la utilización de ganglios de raíz dorsal sometidos a una axotomía y a un aumento de la concentración de calcio en el medio, se observó que la desintegración granular del citoesqueleto de las células es dependiente de la concentración de calcio, puesto que al inhibir los canales de calcio tipo L o proteasas como las calpaínas I y II (enzimas que son dependientes de calcio), ésta es mucho más lenta. Por el contrario, cuando las concentraciones de calcio eran altas $(20 \mathrm{mM})$ y mediante la utilización del ionóforo A23187 (específico para calcio), se observó que el desensamblaje del citoesqueleto y del citoplasma fue más rápido y agresivo (22). 


\section{Sistemas de regulación de calcio}

En estados normales, todas la células presentan mecanismos de regulación para evitar daños que van desde la formación de cristales con radicales como el fosfato, hasta la desintegración y desensamblaje de microtúbulos. Los sistemas de regulación de calcio conocidos son el mecanismo de intercambio o antiporter $\mathrm{Na}^{+} / \mathrm{Ca}^{++}$y la presencia de bombas como la ATPasa de calcio (23).

El sistema de intercambio está asociado a un movimiento de calcio y/o sodio a través de la membrana, en dirección opuesta y dependiente de la distribución de los iones en la membrana. Tal intercambio no requiere ATP y depende del gradiente electroquímico de los iones participantes. Este tipo de transporte puede ser reversible, permitiendo, así, la salida de calcio y la entrada de sodio o viceversa; su estequiometría se relaciona con la toma de un ion de calcio por tres de sodio (1:3).

Aunque se cree que este sistema está en todo tipo de células, se encontró en mayor proporción en las células excitables, actuando en el ingreso o expulsión de los iones durante la estimulación (7).

El otro sistema de regulación es un sistema de transporte activo que depende de la energía del ATP para captar el ion y liberarlo al espacio extracelular o almacenarlo.

La liberación de calcio hacia el exterior de la célula se realiza mediante la ATPasa de calcio de membrana plasmática (PMCA). El almacenamiento del ion en organelos como el retículo endoplásmico (liso y rugoso) o en los calciosomas, también está dado por la ATPasa de calcio de retículo (SERCA) (23). Ambas bombas presentan mucha similitud con respecto a sus sitios activos, a los reguladores endógenos y al funcionamiento y ciclo de la bomba.

\section{Características generales de la ATPasa de calcio}

La ATPasa de calcio, como se dijo anteriormente, es una enzima hidrolítica transmembranal de tipo $P$ y posee dos estados energéticos en su ciclo, E1 (alta afinidad al calcio) y E2 (baja afinidad al calcio) (24).
La proteína consiste en un polipéptido que atraviesa la bicapa lipídica diez veces; las regiones hidrofílicas de la bomba están concentradas en el citoplasma, divididas en tres bloques grandes, además de su $\mathrm{N}$ y $\mathrm{C}$ terminales.

El primer dominio, que está localizado entre las regiones transmembranales 2 y 3 , contiene el sitio fosfatasa y la región de unión a los fosfolípidos. El segundo dominio, que se desprende de las regiones 4 y 5 , es el bloque más grande y contiene el sitio de unión del ATP, el residuo aspartil y una región bisagra, que posiblemente esté involucrada en los cambios de conformación de la enzima.

El tercer dominio corresponde a la región Cterminal, la cual contiene el dominio de unión de la calmodulina y proteínas-cinasa y puede variar de acuerdo con la adición o deleción de pares de bases, lo que permite diferenciar las isoformas. Sobre la membrana, entre las regiones $4,5,6$ y 8 , se halla el sitio de unión al calcio (figura 1).

Una propiedad importante de la ATPasa de calcio es la estimulación por medio de reguladores como la calmodulina (CaM), la cual incrementa la afinidad de la enzima por el calcio. La calmodulina es una proteína soluble en el citoplasma que posee cuatro sitios de unión a calcio. De acuerdo con el número de iones unidos a la proteína, ésta adquiere una conformación estructural que le permite intervenir en varios procesos, como el ensamblaje de microtúbulos, entre otros. La calmodulina, al igual que otros reguladores como

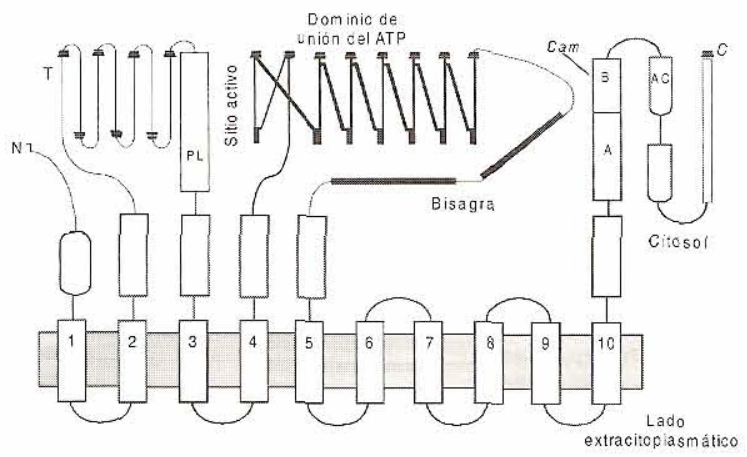

Figura 1. Estructura propuesta para la ATPasa de calcio de membrana plasmática: $\mathrm{N}$ y $\mathrm{C}$ terminales; $\mathrm{PL}$, región de unión a fosfolípidos; CaM, región de unión a la calmodulina; $A C$, región de unión a las proteínas-cinasas. 1-10 dominios transmembranales y entre $4,5,6$ y 8 se ubica el sitio de unión al calcio. Tomado de: Strheler (7) 
los fosfolípidos y las proteínas-cinasa, aumentan la afinidad y la velocidad de reacción enzimática $(7,24,25)$.

Los fosfolípidos y ácidos grasos polinsaturados de cadenas largas aumentan la afinidad de la enzima por el ion más que la calmodulina. Con la utilización de liposomas de diferente composición de fosfolípidos, se observó que la concentración de fosfolípidos ácidos alrededor de la bomba de calcio era suficiente para superar la velocidad máxima de activación de la enzima, comparada con la obtenida con calmodulina $(26,27)$.

La regulación por proteínas-cinasa se realiza mediante la fosforilación de la ATPasa, a través de la unión de la subunidad catalítica de estas proteinas-cinasa a la bomba. Esta fosforilación incrementa tanto la afinidad de la bomba hacia el ion como la velocidad máxima de reacción (24).

Se ha evaluado la interacción de los reguladores de la ATPasa de calcio en microsomas de membrana de eritrocito. Al adicionar proteínascinasa y calmodulina a los microsomas, se observó que la calmodulina puede inhibir la fosforilación de la enzima o no afectar el porcentaje de actividad, al igual que sucede con los fosfolípidos. En el caso de las proteínas-cinasa, este fenómeno se presenta por la cercanía de sus sitios de unión en la región C-terminal de la enzima, demostrando que la calmodulina no posee un efecto aditivo en sus mecanismos de acción con estas proteínas y con los fosfolípidos. Queda por aclarar, entonces, la relación de estos reguladores in vivo de forma tejido-específica y según el tipo de bomba (PMCA o SERCA) $(7,24)$.

\section{Funcionamiento y ciclo}

El modo de funcionamiento de la ATPasa de calcio aceptado hoy en día, se conoce como el modelo de marioneta, puesto que al poseer la proteína una zona bisagra, ésta puede presentar cambios en su conformación que faciliten cumplir con su función de transporte.

En primer lugar, la enzima deja expuestos los sitios de unión al calcio y al ATP, desplazando estos dominios citoplasmáticos por medio de su región bisagra y permitiendo la unión del calcio, así como una subsiguiente hidrólisis de ATP



Figura 2. Ciclo de la ATPasa de calcio. Se muestran los dos estados energéticos dados por la hidrólisis del ATP. E1, unión del ion y del ATP; E2, liberación del calcio y del Pi. El ciclo es reversible en presencia constante de calcio y ATP. Tomado de: McLennan, Toyofuku, Lytton (28).

$(\mathrm{ADP}+\mathrm{Pi})$, la fosforilación del residuo aspartil y el inicio de un estado de alta energía y afinidad E1. La enzima sufre una serie de cambios de conformación posteriores, dando lugar a la formación de un poro para la expulsión del ion. El ion es liberado, el fósforo inorgánico se separa por hidrólisis, entrando en el estado de baja afinidad E2 y, finalmente, ocurre de nuevo el desplazamiento de los dominios a su estado original E2--->E1 (figura 2) (28-31).

\section{Aspectos moleculares de la ATPasa de calcio}

Como se dijo anteriormente, la ATPasa de calcio encontrada en la membrana plasmática y la hallada en la membrana de retículo sarcoendoplásmico, se denominan PMCA y SERCA, respectivamente. Ambos tipos de bombas cumplen la función de regulación del ion en la célula y presentan homologías en su sitio activo y de regulación, pero difieren en sus aspectos moleculares.

Las bombas de membrana plasmática (PMCA) (cuadro 1), con un peso molecular entre 130 a $140 \mathrm{KD}(7)$, toman el calcio del citoplasma y lo liberan al espacio extracitoplasmático cuando la concentración interna del ion es mayor que 0,1 $\mu \mathrm{M}$ (32). Fue descubierta en eritrocitos, en donde parece ser el sistema de regulación de calcio más importante (7). Las bombas de membrana plasmática son codificadas por cuatro genes diferentes en humano y en rata. De acuerdo con la inclusión o deleción de pares de bases en sitios específicos, como la región $\mathrm{C}$ terminal en la zona de unión a la $\mathrm{CaM}$, se generan varias isoformas, PMCA1, PMCA2, PMCA3 Y PMCA4 (27). 
Cuadro 1. Propiedades de la bomba de calcio de membrana plasmática (PMCA).

\begin{tabular}{ll}
\hline Distribución & Todas las células animales \\
Peso molecular & $\begin{array}{l}\text { Aproximadamente } 134.000 \mathrm{Da} \\
\text { Clase } \mathrm{P} \\
\text { Tipo }\end{array}$ \\
$\begin{array}{l}\text { Vanadato, lantano } \\
\text { Mecanismo de acción }\end{array}$ & $\begin{array}{l}\text { Formación de un intermedio } \\
\text { aspartil-fosfato }\end{array}$ \\
Estequiometría Ca/ATP & $1: 1$ \\
Afinidad al calcio & $\begin{array}{l}10-20 \mu \mathrm{M} \text { en estado de baja } \\
\text { afinidad } \\
0,5 \mu \mathrm{M} \text { o menos en estado de } \\
\text { alta afinidad } \\
\text { Calmodulina que induce a una } \\
\text { transición de alta afinidad }\end{array}$ \\
\hline
\end{tabular}

Tomado de: Carafoli, Staufer (26).

Entre las isoformas de PMCA, se presentan altamente conservadas las secuencias de los dominios esenciales de las unidades catalíticas y de transporte de la enzima; sin embargo, se presentan divergencias en las secuencias debido, quizá, a la especialización de la enzima y su adaptación de acuerdo con las necesidades fisiológicas del tejido. Esto se observó en estudios realizados por medio del análisis de Northern blot, en los cuales se encontró que el mARN de la isoforma PMCA1 en rata, se expresa en mayor cantidad en músculo esquelético, mientras el de PMCA4 se encontró en mayor proporción en eritrocito $(7,26,33)$.

Es de resaltar, también, que dentro de cada isoforma se generan otras variaciones que son codificadas por los mismos genes durante la transcripción en el proceso de splicing. Estas isoformas contienen las regiones variables de unión a fosfolípidos, calmodulina y proteínascinasa, lo que se ajustaría a las necesidades del tejido (26).

En el caso de las bombas de retículo (SERCA), con un peso molecular entre 115 a $120 \mathrm{KD}$ (27), el ion libre en el citoplasma es captado y liberado en el lumen del retículo para su almacenamiento; posteriormente, el ion es liberado por la misma enzima. Esta enzima fue descubierta en músculo esquelético, donde la ATPasa de calcio cumple un papel importante en la modulación del ion para la contracción muscular (33).
De la ATPasa de retículo endoplásmico, SERCA, se conocen tres genes que codifican tres isoformas SERCA1, SERCA2, SERCA3. Con estas isoformas sucede lo mismo que con las bombas de membrana plasmática, siendo tejidodependientes y presentando variaciones por splicing alternativo.

Por el mismo análisis (Northern blot), se detectó que el mARN de SERCA1 se encuentra en gran cantidad en músculo esquelético rápido, el de SERCA2 se presenta en gran cantidad en músculo esquelético lento, cardiaco y liso, cerebro, mucosa estomacal, hígado y riñón, entre otros. En el caso de la isoforma SERCA3, se encuentra en gran cantidad principalmente en intestino grueso y bazo, en cantidades moderadas en cerebro, estómago, útero y músculo esquelético, y en muy baja cantidad en el resto de los tejidos.

SERCA también es regulada por fosfolípidos y proteínas-cinasa y se ha encontrado especificidad por estos reguladores, por ejemplo SERCA 1 y 2 poseen sitios de unión a fosfolípidos mientras que SERCA 3 presenta gran sensibilidad a la fosforilación de las proteínas-cinasa dependientes de AMPc (27).

En tejido nervioso se ha descubierto que el retículo endoplásmico también almacena calcio, captando el ion del citoplasma e introduciéndolo al lumen del retículo por la misma enzima (12). Aunque no hay muchos estudios relacionados con SERCA en tejido nervioso, se cree que la isoforma que se expresa en mayor proporción es SERCA2 $(24,26,32)$.

\section{ATPasa de calcio en tejido nervioso}

Por diferentes técnicas, se ha purificado la ATPasa de calcio en tejido nervioso y en otros tejidos. En cerebro de bovino, por ejemplo, se logró secuenciar completamente el ADN que codifica para la bomba de membrana plasmática y se lograron diferenciar las isoformas PMCA1 y PMCA2 (32,34).

Con la elaboración de vesículas o microsomas de homogenizados de diferentes tejidos y zonas del cerebro $(25,35)$ o mediante la inmunodetección con anticuerpos dirigidos contra el dominio citoplasmático de la ATPasa de calcio, ésta se 
ha ubicado en el sistema nervioso central (SNC), en la membrana plasmática de tejido ependimario y plexo coroideo $(36,37)$, en corteza cerebral, en cerebelo en las células de Purkinje $(38,39)$ y en nervio óptico.

Para corroborar la especificidad de las isoformas y la presencia de la ATPasa de calcio en diferentes zonas del cerebro de rata, se han utilizado anticuerpos dirigidos contra las isoformas (PMCA1, PMCA2, PMCA3) de la bomba de calcio de membrana plasmática, demostrando que, aunque las tres isoformas se encuentran y se expresan en todo el tejido nervioso, hay variabilidad en la marcación entre las diferentes zonas del cerebro.

Para PMCA1, se presentó mayor marcación en hipocampo, hipotálamo, bulbo olfatorio y regiones del cerebro medio. La marcación para PMCA2 fue específica en zonas de cerebelo, en soma y dendritas de células de Purkinje. Con el anticuerpo para PMCA3, la marcación fue intensa en zonas como el cuarto ventrículo, cerebelo y plexo coroideo (40).

En el sistema nervioso periférico (SNP), se ha detectado la enzima en membrana plasmática de terminaciones sinápticas en placas neuromotoras y en axolema de fibras de nervio periférico $(11,17,41,42)$.

Por medio de la elaboración de microsomas de nervios como el ciático o mesentérico y con la utilización de técnicas bioquímicas como la medición del fósforo inorgánico liberado por la hidrólisis de ATP para determinar el porcentaje de actividad de la enzima (12), se determinó la existencia de bombas que presentaban diferentes afinidades al calcio de acuerdo con su concentración en el medio de incubación (42-44). Mediante estas condiciones se distinguen dos tipos de bombas dependientes de la concentración del ion dentro del citoplasma.

A las bombas que son activadas en presencia de concentraciones de calcio, que oscilan entre $0,1 \mathrm{mM}$ y $1 \mathrm{mM}$, se les denomina bombas de alta afinidad y poseen características como su sensibilidad a la calmodulina y su insensibilidad al vanadato (inhibidor específico para las bombas de tipo P). Las otras bombas son activadas en concentraciones de calcio entre $5 \mathrm{mM}$ y $12 \mathrm{mM}$ y son insensibles a la calmodulina pero dependientes del magnesio, inhibidas por vanadato y denominadas de baja afinidad o Ecto-ATPasas $(25,43)$. Esto se ha comprobado también en terminales sinápticas (41), hígado (42), papilas gustativas (43), placas neuromotoras (44) y nervio periférico (45).

Mediante citoquímica enzimática, se ha detectado la actividad de algunas enzimas hidrolíticas en la mayoría de los tejidos. Esta técnica se basa en la captura del producto de la hidrólisis enzimática con un metal pesado en condiciones óptimas de temperatura y $\mathrm{pH}$ para la activación enzimática.

Esta técnica ha sido utilizada para algunas enzimas, incluida la ATPasa de calcio, en la cual se requiere tanto de un sustrato como de un activador específico para la enzima. En el caso de la bomba de calcio estos son el ATP y el calcio, respectivamente; la unión de éstos a sus sitios activos estimula la enzima para que ésta hidrolice el ATP en ADP y Pi, los cuales forman un complejo denominado producto de reacción primario (PRP).

Además, se requiere de un agente de captura como el plomo o el cerio, que reaccionan con el fósforo inorgánico $(\mathrm{Pi})$, formando el producto de reacción final (PRF), y conforman un complejo insoluble y electrodenso que permite ser visualizado por microscopía electrónica. De esta forma, se ha detectado la actividad enzimática de la bomba de calcio en la membrana plasmática de células de glía periférica (células de Schwann), en el axolema, en los organelos y en los microtúbulos de fibras mielínicas y amielínicas de nervio periférico (45-49).

En sistema nervioso central, la actividad se ha visualizado sobre la membrana externa de la mielina compacta, el axolema, la membrana plasmática de astrocitos y en células ependimarios. En ganglio de raíz dorsal también se ha detectado sobre la membrana de células satélites y sobre la membrana de neuronas.

También se ha detectado la actividad de la enzima en la membrana de células endoteliales de los vasos sanguíneos presentes en el tejido nervioso $(11,42,48)$. En otros tejidos se ha encontrado sobre la membrana de hepatocitos, eritrocitos y túbulos 
proximales y distales del riñón $(49,50)$. Mediante esta técnica, también se ha confirmado la diferencia de afinidades de la enzima al calcio, demostrando la existencia de bombas de alta y baja afinidad $(11,45)$.

Aunque hay avances en la literatura sobre la función e importancia del calcio en la degeneración de nervio periférico por lesiones como axotomía o aplastamiento, no hay muchos estudios sobre la regulación del ion por medio de la ATPasa de calcio y su actividad tanto en nervio normal como lesionado.

Como se ha visto, en la mayoría de las células, la ATPasa de calcio es uno de los sistemas de regulación del ion más importante. Sin embargo, aún quedan por comprender muchos aspectos de su fisiología y su papel en la regulación del calcio y en los procesos de degeneración y regeneración de nervio periférico. Por esta razón, entre las proyecciones de investigación para la ATPasa de calcio en nuestros laboratorios, está determinar el comportamiento de la actividad de la enzima por citoquímica enzimática en diferentes tiempos de denervación y regeneración, en los muñones proximales y distales de nervios transectados en modelos animales.

\section{Referencias}

1. Carafoli E. The $\mathrm{Ca}^{2+}$-ATPase of the plasma membrane. In: Cellular calcium. New York: Oxford University Press; 1991. p.299-312.

2. Pietrobon D, Di Virgilio F, Pozzan T. Structural and functional aspects of calcium homeostasis in eukaryotic cells. Eur J Biochem 1990;193:599-622.

3. Vorherr T, James P, Krebs J, Enyedi A, McCormick D, Penniston JT, et al. Interaction of calmodulin with the calmodulin binding domain of the plasma membrane $\mathrm{Ca}^{2+}$ pump. Biochemistry 1990;29:355-65.

4. Cheung $W$ Y. Calmodulin and its activation by cadmium ion. Ann N Y Acad Sci 1988;522:74-87.

5. Means A, Dedman J. Calmodulin, an intracellular calcium receptor. Nature 1980;265:73-7.

6. Llinás R. The intrinsic electrophysiological properties of mammalian neurons: insights into central nervous system function. Science 1988;268:1654-64.

7. Strehler E. Plasma membrane $\mathrm{Ca}^{2+}$ pump and $\mathrm{Na}^{+} / \mathrm{Ca}^{2+}$ exchangers. Semin Cell Biol 1990;1:283-95.

8. Blaustein P. Sodium calcium exchange: its role in the regulation of cell calcium. In: Carafoli E, editor. Membrane transport of calcium. New York: Academic Press; 1982. p.217-36.

9. Carafoli E. The transport of calcium across the inner membrane of mitochondria. In: Carafoli E, editor. Membrane transport of calcium. New York: Academic Press; 1982. p.109-35.

10. Carafoli E, Garcia-Martin E, Guerini D. The plasma membrane pump: recent developments and future perspectives. Experientia 1996;52:1091-100.

11. Maxwell W L, Mccreath B J, Graham D. Cytochemical evidence for redistribution of membrane pump calcium-ATPase and ecto-Ca-ATPase activity, and calcium influx in myelinated nerves fibres of the optic nerve after stretch injury. J Neurocytol 1995;24:925-42.

12. Henkart M, Reese T, Brinley F. Endoplasmic reticulum sequestres calcium in the squid giant axon. Science 1978; 202:1300-3.

13. Finkbeiner S. Glial calcium. Glia 1993;9:83-104.

14. Carafoli E. La señal del calcio. Innovacion y Ciencia 1986;78:28-37.

15. Mata M, Staple J, Fink DJ. Ultrastructural distribution of calcium within neurons: an oxalate pyroantimonate study. Histochemistry 1987;87:339-49.

16. Ochs S, Worth R, Chan S. Calcium requirement for axoplasmic transport in mammalian nerve. Nature 1977; 270:748-50.

17. Ochs S, Brimijoin S. Axonal transport. In: Peripheral Neurophathology. Third Edition. Philadelphia: W.B. Saunders Co., 1993. p.335-9.

18. Augustine G, Charlton M, Smith S. Calcium action in synaptic transmitter release. Annu Rev Neurosci 1987; 10:633-93.

19. Parson JT, Churn SB, De Loreto RJ. Ischemia-induced inhibition of calcium uptake into rat brain microsomes mediated by $\mathrm{Mg}^{2+} / \mathrm{Ca}^{2+}$ ATPase. J Neurochem 1997;68:1124-34.

20. Lopachin R Jr, Lopachine V, Saubermann A. Effects of axotomy on distribution and concentration of elements in rat sciatic nerve. J Neurochem 1990;54:32032.

21. Griffin J, Hoffman P. Degeneration and regeneration in the peripheral nervous system. En: Dick P, editor. Peripheral neuropathy. Third edition. Philadelphia: W.B. Saunders Co.; 1993. p.76-361.

22. George E, Glass J, Griffin J. Axotomy-induced axonal degeneration is mediated by calcium influx through ionspecific channels. J Neurosci 1995;15:66445-52.

23. Carafoli E. Intracellular calcium homeostasis. Annu Rev Biochem 1987;56:395-433.

24. Wuytack F, Raeymaekers L. The $\mathrm{Ca}^{2+}$-transport ATPases from the plasma membrane. J Bioenerg Biomembr 1992;24:285-300. 
25. Kostka P, Barnett W, Kwan C. Identification and characterisation of high-affinity $\mathrm{Ca}^{2+}$-ATPase associated with axonal plasma membranes of dog mesenteric nerves. Neurochem Res 1990;15:833-41.

26. Carafoli E, Stauffer T. The plasma membrane calcium pump: functional domains, regulation of the activity, and tissue specificity of isoform expression. J Neurobiol 1994;25:312-24.

27. Grover A, Khan I. Calcium pump isoforms: diversity, selectivity and plasticity. Cell Calcium 1992;13:9-17.

28. Maclennan D, Toyofuku T, Lytonn J. Structure-function relationships in sarcoplasmic or endoplasmic reticulum type $\mathrm{Ca}^{2+}$ pumps. Ann N Y Acad Sci 1992;671:1-10.

29. Carafoli E. The $\mathrm{Ca}^{2+}$ pump of the plasma membrane. $J$ Biol Chem 1992;267:2115-8.

30. Wang K, Villalobo A, Roufogalis B. The plasma membrane calcium pump: a multiregulated transporter. Trends Cell Biol 1992;2:46-52.

31. Shull G, And Greeb J. Molecular cloning of two isoforms of the plasma membrane $\mathrm{Ca}^{2+}$ transporting ATPase from rat brain: structural and functional domains exhibit similarity to $\mathrm{Na}^{+} / \mathrm{K}^{+}$and other cation transport ATPases. J Biol Chem 1988;263:8646-57.

32. West J. Bases fisiológicas de la práctica médica. 12 edición. Baltimore: William \& Wilkins; 1991.

33. Verma A, Filoteo A, Stanford D, Wieben E, Penniston J. Complete primary structure of a human plasma membrane $\mathrm{Ca}^{2+}$ pump. J Biol Chem 1988;263: 14152-9.

34. Gill D, Grollman E, Kohn L. Calcium transport mechanisms in membrane vesicles from Guinea pig brain synaptosomes. J Biol Chem 1981;256:184-92.

35. Nagano M, Fujioka A, Mori S. Cytochemical demostration of $\mathrm{Ca}^{2+}$-ATPase in the choroid plexus of the third ventricle. Acta Histochem Cytochem 1991;24: 7783.

36. Borke J, Caride A, Yaksh T, Penniston J, Kumar R. Cerebrospinal fluid calcium homeostasis: evidence for plasma membrane $\mathrm{Ca}^{2+}$-pump in mammalian choroid plexus. Brain Res 1989;189:355-60.

37. De Talamoni N, Smith CA, Wasserman RH, Fullmer CS, Penniston JT. Immunocytochemical localization of the plasma membrane calcium pump, calbindin-D28K and parvalbumin in Purkinje cells of avian and mammalian cerebellum. Proc Natl Acad Scien USA 1993;90:1194953.
38. Hillman DE, Chen S, Bing R, Penniston Jt, Llinas R. Ultraestructural localisation of the plasmalemmal calcium pump in cerebellar neurons. Neuroscience 1996;72:31524.

39. Stuffer T, Guerini D, Celio M, Carafoli E. Immunolocalization of the plasma membrane $\mathrm{Ca}^{2+}$ pump isoforms in the rat brain. Brain Res 1997;748:21-9.

40. Mata M, Staple J, Fink D. Cytochemical localization of $\mathrm{Ca}^{2+}$-ATPase activity in peripheral nerve. Brain Res 1988; 445:47-54.

41. Korte K, Frihofer D, Rahmann H. Cytochemical localization of high affinity $\mathrm{Ca}^{2+}$ ATPase activity in synaptic terminals. J Histochem Cytochem 1990;38:895-900.

42. $\mathrm{Lin} \mathrm{S}$. The rat liver plasma membrane high affinity $\left(\mathrm{Ca}^{2+}-\right.$ $\mathrm{Mg}^{2+}$ )- ATPase is not a calcium pump. J Biol Chem 1985; 260:10976-80.

43. Barry M. Ecto-calcium-dependent ATPase activity of mammalian taste bud cells. J Histochem Cytochem 1992; 42:1919-28.

44. Papas G, Kriho V. Fine structural localisation of $\mathrm{Ca}^{2+}$ ATPasa activity at frog neuromuscular junction. J Neurocytol 1988;17:417-23.

45. Mizutani $\mathrm{Y}$, et al. Fine localisation of low and high calcium dependent ATPase activities in the rat sciatic nerve. Brain Res 1995;442:693.

46. Lewis RP. Metal precipitation methods for hidrolitic enzymes. In: Staining methods for sections material: practical methods. En: Electron microscopy. Oxford: North Holland Publishing Company; 1977.

47. Mata M, Fink DJ. $\mathrm{Ca}^{2+}$ ATPase in the central nervous system: an EM cytochemical study. J Histochem Cytochem 1989;37:971-80.

48. Ando T, Fujimoto $\mathrm{K}$, Mayahara, et al. A new onestep method for the histochemistry and cytochemistry of $\mathrm{Ca}^{2+}$-ATPase activity. Acta Histochem Cytochem 1981; 14:705-26.

49. Caldas, ML, Wasserman M. Estandarización de un método citoquímico con microscopía electrónica para detectar la actividad enzimática en células completas. Biomédica 1995;15:197-205.

50. Velandia ML. Determinación ultraestructural de la actividad de la ATPasa de calcio en nervio ciático normal y denervado crónicamente en un modelo de regeneración mediante implante de células de Schwann autólogas de rata adulta (tesis). Bogotá, D.C.: Universidad Distrital; 2000. 